\title{
THE INFLUENCE OF THERMAL CONDUCTION ON THE FIELD DEPENDENCE IN SPIN-LATTICE RELAXATION EXPERIMENTS
}

\author{
J. FLOKSTRA, G. J. GERRITSMA and L. C. van der MAREL \\ Technische Hogeschool Twente, Afdeling der Technische Natuurkunde, Enschede, The Netherlands
}

Received 10 May 1977

\begin{abstract}
It is demonstrated that heat transfer in magnetic samples and the surrounding liquid helium leads to a field dependence of the observed relaxation time in low-frequency relaxation measurements which deviates strongly from that of the intrinsic spin-lattice relaxation.
\end{abstract}

\section{Introduction}

The dispersion and absorption curves, obtained in low-frequency relaxation experiments on paramagnetic crystals in a liquid-helium bath having a temperature above the helium transition temperature $T_{\lambda}$, strongly deviate in many cases from the shape of the Casimir and Du Pré curves. The deviations are considerably smaller for $T<T_{\lambda}$. In a previous paper [1] we introduced a thermodynamic model for the relaxation (the thermal conduction model) involving the heat properties of the environment of the crystal. With this model it was possible to explain satisfactorily the shape of the susceptibility curves for $T>T_{\lambda}$ as well as for $T<T_{\lambda}$. Assuming a direct process for the spin-lattice relaxation $\left(\tau_{\mathrm{s}} \propto T^{-1}\right)$, it was calculated that the temperature dependence of the time constant $\tau_{\text {abs }}$ obtained from the absorption curve in the conventional way may differ greatly from $T^{-1}$. It was found that $\tau_{\text {abs }} \propto T^{-\alpha}$ with $1<\alpha \lesssim 6$, the large values for $\alpha$ being due to bad thermal conduction in the lattice or the surrounding medium.

In the present paper the influence of the abovementioned thermal effects on the field dependence of the relaxation time constant is investigated. The field dependence of $\tau_{\mathrm{abs}}$ and of the parameter $d$, which describes the deviations of the measured absorption curve with respect to the absorption curve according to the Casimir and Du Pré theory, shows a number of characteristic features for many compounds. As a typical example experimental results on $\mathrm{YbCl}_{3} \cdot 6 \mathrm{H}_{2} \mathrm{O}$ taken from ref. 2 are presented (fig. 1). Corrections were made for the anisotropy of the relaxation time constant. It is seen that the deviation parameter $d$ in the strong-field range increases rapidly with increasing field and that the slope of the $\tau$ vs. $H$ curve may change in sign in this field range. The radius of the sample has a great influence on $\tau_{\mathrm{abs}}$ and $d$.

Using the thermal conduction model we calculate in this paper that the experimental results $\tau_{\text {abs }}(H)$ and $d(H)$ at strong fields may be due to bad heat conduction in the lattice and the surrounding medium.

\section{The thermal conduction model and the calculations}

A diagram of the thermal conduction model is given in fig. 2. The spin system (specific heat $C_{\mathrm{H}}$ ) is coupled to the lattice $\left(C_{\mathrm{L}}\right.$, thermal conductivity $\left.\lambda_{\mathrm{L}}\right)$ by the heat transfer coefficient $\alpha$. The thermal contact resistance between lattice and bath $\left(C_{\mathrm{g}}, \lambda_{\mathrm{g}}\right)$ is denoted by $R_{\mathrm{K}}$. The expression for the susceptibility of a sample with a characteristic dimension $r_{0}$ is given in ref. 1. Apart from scaling factors the dispersion and absorption are fully determined by the dimensionless parameters $D$ and $R$ (ratios of specific heats) and $P$, $Q$ and $S$ (ratios of coefficients of heat transfer). The field-dependent parameters are $C_{\mathrm{H}}$ and $\alpha\left(=C_{\mathrm{H}} / \tau_{\mathrm{s}}\right)$ 

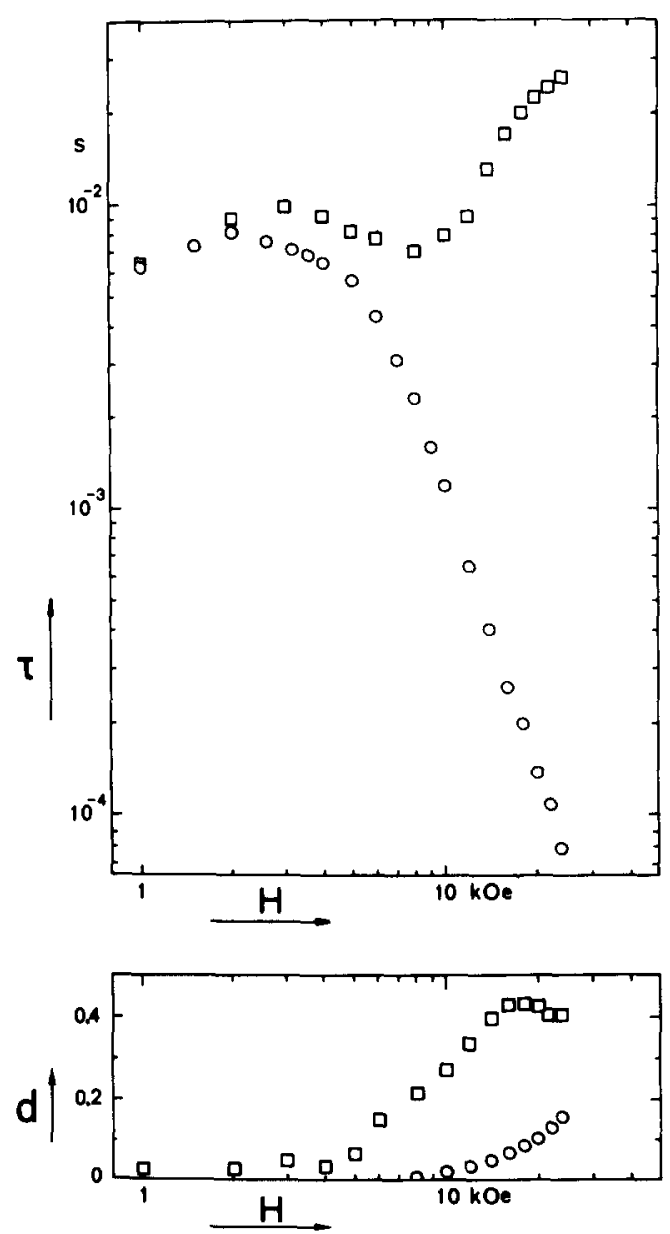

Fig. 1. Relaxation time constants and deviation parameters of $\mathrm{YbCl}_{3} \cdot 6 \mathrm{H}_{2} \mathrm{O}$ as a function of the magnetic field at $4.2 \mathrm{~K}$. The data are taken from ref. $2.0=$ powdered sample; $\square=$ single crystal (orientation angle $\theta=30^{\circ}$ ). For the powdered sample we subtracted 0.15 from the value of $d$ and we fitted the $\tau_{\text {abs }}(H)$ curve to that of the single crystal at $1 \mathrm{kOe}$ in order to compensate for the anisotropy effect.

and therefore also $D, R$ and $P$. It is usual to write $C_{\mathrm{H}}$ as

$C_{\mathrm{H}}=\frac{b}{T^{2}}+\mu_{0} C \frac{H^{2}}{T^{2}}$

where $C$ is the Curie constant and $b$ a constant related to an internal field by $b=\mu_{0} C H_{\text {int }}^{2}$. If $D_{0}$ and $R_{0}$ denote the values of $D$ and $R$ at $H=0$ then

$D=C_{\mathrm{H}} / C_{\mathrm{L}}=D_{0}\left[1+H^{2} / H_{\mathrm{int}}^{2}\right]$,

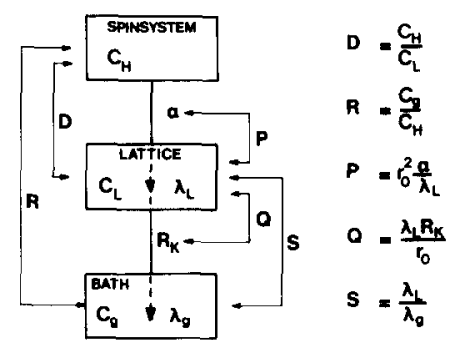

Fig. 2. The thermal conduction model and the dimensionless parameters.

$R=C_{\mathrm{g}} / C_{\mathrm{H}}=R_{0}\left[1+H^{2} / H_{\text {int }}^{2}\right]^{-1}$.

It is assumed that the spin-lattice relaxation time $\tau_{\mathrm{s}}$ is a constant $\tau_{s_{0}}$ at weak magnetic fields and that it is determined by the direct process at strong fields so that

$\tau_{\mathrm{s}}^{-1}=\tau_{\mathrm{s}_{0}}^{-1}+A H^{4} T$.

This can be also written as

$\tau_{\mathrm{s}}=\tau_{\mathrm{s}_{0}}\left[1+\epsilon H^{4} / H_{\mathrm{int}}^{4}\right]^{-1}$,

where $\epsilon=A H_{\text {int }}^{4} T \tau_{\mathrm{s}_{0}}$ is independent of the field. For $P$ the following expression can be derived:

$P=P_{0}\left[1+H^{2} / H_{\text {int }}^{2}\right]\left[1+\epsilon H^{4} / H_{\text {int }}^{4}\right]$,

where $P_{0}$ is the value of $P$ at $H=0$. The dimensionless parameters $P$ and $D$ increase rapidly with increasing field for $H>H_{\text {int }}$, while $R$ decreases. In ref. 1 it is shown that a large magnitude of $P$ and $D$ results in strongly broadened absorption curves for which $\tau_{\text {abs }}$ may deviate considerably from $\tau_{\mathrm{s}}$, so these phenomena can also be expected in the present calculations.

The field dependence of $\tau_{\mathrm{abs}}$ and $d$ was calculated for various values of the parameters. As a typical example the results for $D_{0}=1, R_{0}=1000, S=10$, $Q=0$ and $P=10,1,0.1,0.01$, respectively, are presented in fig. 3 . Hence, the thermal resistance between crystal and bath is taken as zero. The arbitrary value of 1 was taken for $\epsilon$, so that the direct process becomes effective at $H=H_{\text {int }}$. The different values for $P_{0}$ represent a variation of the crystal size. The values for the parameters correspond to realistic, experimental circumstances, although they do not apply to a particular compound. It is seen that the 

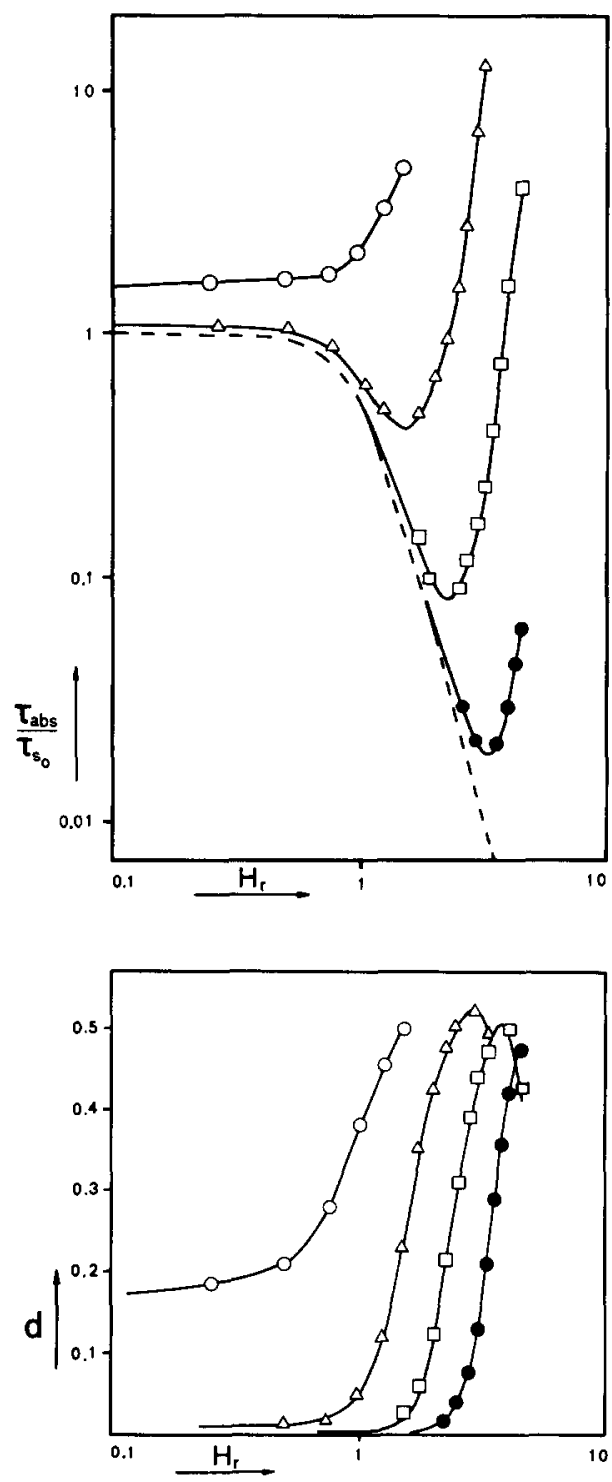

Fig. 3. The field dependence of $\tau_{\text {abs }}$ and $d$ of the calculated susceptibility curves. $H_{\mathrm{r}}=H / H_{\text {int }} \cdot D_{0}=1, R_{0}=1000$, $S=10, Q=0, \epsilon=1.0=P_{0}=10 ; \triangle=P_{0}=1 ; \square=P_{0}=0.1$; $\bullet=P_{0}=0.01$. The dashed curve corresponds to the spinlattice relaxation time $\tau_{\mathbf{s}}$.

smaller the value of $P_{0}$, the larger the range for which $\tau_{\text {abs }}$ follows the spin-lattice relaxation time $\tau_{\mathrm{s}}(H)$. At a certain field $\tau_{\mathrm{abs}}(H)$ reaches a minimum value and then increases rapidly with increasing field. For $P_{0}=10$, however, $\tau_{\text {abs }}$ is considerably larger than $\tau_{\mathrm{s}}$ even for $H<H_{\text {int }}$ and the minimum in $\tau_{\mathrm{abs}}(H)$ does not occur. The deviation parameter becomes very large in the field range around the minimum in the $\tau_{\text {abs }}(H)$ curve. At strong fields $d$ again decreases.

\section{Discussion}

The calculated curves in fig. 3 show a great resemblance to the experimental results in fig. 1. Both figures exhibit a considerable size effect. The parameter $P$ is proportional to the square of the radius of the crystal, so small crystals (e.g. powdered samples) lead to time constants almost equal to $\tau_{\mathrm{s}}$ while large crystals give large deviations between $\tau_{\mathrm{abs}}$ and $\tau_{\mathrm{s}}$. The shape of the calculated curves for strong fields is qualitatively in agreement with the characteristic shape of $\tau_{\mathrm{abs}}(H)$ and $d(H)$ of $\mathrm{YbCl}_{3} \cdot 6 \mathrm{H}_{2} \mathrm{O}$. The $\tau(H)$ curve of the powdered sample does not show a minimum at strong fields and also the deviation parameter is still small. It may be expected, however, that at still stronger fields a minimum will occur while $d$ then reaches a large value.

At weak magnetic fields the calculated susceptibility curves are in agreement with the Casimir and Du Pré theory. In this case $\tau_{\mathrm{abs}}$ is equal to $\tau_{\mathrm{s}}$ and $d=0$. Deviations occur when $P$ has a large value. The calculated susceptibility curves are separated into two parts at very strong magnetic fields. The high-frequency part corresponds to the relaxation to the isolated lattice but when $C_{\mathrm{H}} \gg C_{\mathrm{L}}$ the effect on the susceptibility becomes very small. The low-frequency part represents the relaxation due to thermal conduction effects. (In the example of fig. 3 the rate-determining process in the energy transport is the thermal conduction in the liquid helium because $\lambda_{L} \gg \lambda_{g}$.) This relaxation process dominates in the susceptibility when $C_{\mathrm{H}} \gg C_{\mathrm{L}}$. The deviation parameter is then rather small. In the transition region between the weak and strong-field behaviour a maximum in the $d(H)$ curve occurs and $\tau_{\text {abs }}$ already deviates considerably from $\tau_{\text {s. }}$. Hence, $\tau_{\text {abs }}$ contains hardly any information about the spin-lattice relaxation for field values which are larger than the field where the deviation parameter increases rapidly. On the contrary, it is a time constant which characterizes the thermal transport in lattice or bath.

In our opinion it is unlikely that the time constants for the single crystal of $\mathrm{YbCl}_{3} \cdot 6 \mathrm{H}_{2} \mathrm{O}$ represent a spin-lattice relaxation process for $H>2 \mathrm{kOe}$. It is necessary to study large single crystals of magnetically 
concentrated compounds with the so-called "liquidvacuum" method [3] in order to be able to separate the effect of thermal conduction from the intrinsic spin-lattice relaxation phenomena.

\section{References}

[1] J. Flokstra, G. J. Gerritsma, G. A. Hartemink and L. C. van der Marel, Physica 77 (1974) 99.

[2] J. Soeteman, L. Bevaart and A. J. van Duyneveldt, Physica 74 (1974) 126.

[3] J. Flokstra, G. J. Gerritsma, C. L. M. Pouw and A. J. van Duyneveldt, Physica 92B (1977) 339. 